\title{
Le système endocannabinoïde central
}

> Le système dit «endocannabinoïde» pourrait tout à fait être dénommé autrement, tant il Laurent Venance, Raphael Maldonado, Olivier Manzoni apparaît plus large et plus complexe que celui finalement relativement restreint des cannabinoïdes exogènes. Jusqu'à ce jour, les études sur les cannabinoïdes, puis sur les endocannabinoïdes, vont de rebondissements en remise en cause de concepts établis. Ainsi, on attendait un alcaloïde comme principe actif du cannabis, pour finalement identifier un terpénoïde $\left(\Delta^{9}\right.$-tétrahydrocannabinol). Étant donné sa nature chimique, ce dernier devait théoriquement agir de manière indépendante de tout récepteur; or, deux récepteurs ont déjà été clonés (CB1 et $\mathrm{CB} 2)$ et l'existence d'autres récepteurs est très probable. La recherche des ligands endogènes a réservé une surprise de taille aux scientifiques: ce sont des composés lipidiques (cinq ont été identifiés à ce jour); à peine deux d'entre eux avaient-ils été classés comme des neurotransmetteurs à part entière que leur capacité d'agir comme des messagers neuronaux rétrogrades a été mise en évidence. Enfin, les endocannabinoïdes activent des récepteurs, tels que les vanilloïdes, appartenant à d'autres familles, et peuvent donc moduler de manière extrêmement fine de multiples voies de transduction du signal et de l'information, étendant des perspectives thérapeutiques déjà très prometteuses. <

Au milieu du XIX ${ }^{e}$ siècle, les chimistes recherchant le principe actif de la plante Cannabis sativa explorèrent, par analogie avec d'autres phytocomposés psychoactifs identifiés (morphine et cocaïne), la piste d'un alcaloïde. Celle-ci égara les recherches pendant plus de 100 ans, puisqu'il faut attendre 1964 et les travaux du groupe de Raphael Mechoulam pour obtenir la purification et

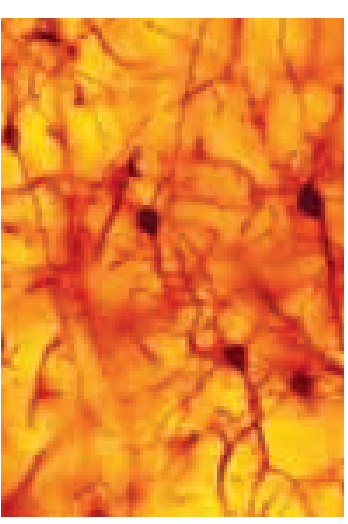

l'identification du principe actif du cannabis: le $\Delta^{9}$-tétrahydrocannabinol ( $\left.\Delta^{9}-T H C\right)$, qui s'avéra finalement être un terpénoïde (Figure 1). La chimie des cannabinoïdes (CB) connut un fulgurant essor tandis que, paradoxalement, leurs mécanismes d'actions moléculaires demeuraient flous. En effet, le $\Delta^{9}$-THC était supposé appartenir au groupe des lipides bioactifs, et donc avoir des mécanismes d'action comparables à ceux des anesthésiques et solvants, c'est-à-dire indépendants d'une liaison à un récepteur. Or, la stéréospécificité de l'action du $\Delta^{9}(-)$-THC fut mise en évidence, favorisant l'hypothèse de l'existence d'un récepteur pouvant lier ces molécules psychoactives. En 1990, le premier récepteur des $C B(C B 1)$ fut cloné dans le système nerveux central (SNC) [1], ouvrant la voie à la recherche d'un système endocannabinoïde (endoCB). En effet, s'il existait un récepteur d'un composé exogène d'origine végétale $\left(\Delta^{9}\right.$ $T H C$ ), une ou des molécules endogènes agonistes de ce récepteur existaient probablement (de manière similaire 
au trio opiacés/peptides opioïdes endogènes/récepteurs opioïdes).

Là encore, de nombreuses pistes infructueuses furent

explorées: aucun des neurotransmetteurs, hormones ou diverses substances biologiquement actives ne se lièrent au récepteur CBl. La nature chimique de l'agoniste endogène constitua, encore une fois, le principal frein à son identification: il s'agissait d'un acide gras; or, une telle molécule n'était pas le meilleur candidat pour être agoniste d'un récepteur. De plus, sa purification constituait un tour de force technique, qui fut également réalisé par le groupe de R. Mechoulam [2]. Cet endocannabinoïde (endoCB), l'arachidonoyléthanolamide, fut dénommé anandamide (AEA), fusion des mots ananda, signifiant bonheur suprême en sanskrit, et d' «amide», du fait de sa structure chimique. Rapidement, un second récepteur des CB (CB2) fut cloné à partir de rate de rat [3, 9], puis d'autres molécules endogènes pouvant se lier aux récepteurs $C B$ furent identifiées. La mise en évidence d'un véritable système endoCB était en cours.

\section{Le système endocannabinoïde}

II est composé de récepteurs, d'endoCB et des systèmes de synthèse, transport et dégradation de ceux-ci [4]. Un endoCB est une molécule endogène capable de se lier à un récepteur cannabinoïde (identifié grâce à des $C B$ exogènes synthétiques) et d'activer les voies de transduction du signal auxquelles est couplé le récepteur. Cinq ont été identifiés: trois anandamides, le 2arachidonoyl glycérol (2-AG) et le 2-AG éther (Figure 1). Les études, qui ont principalement porté sur le «vrai » anandamide (AEA) et le 2-AG, montrent qu'ils possèdent toutes les caractéristiques des neurotransmetteurs «classiques», à une exception près, leur mode de stockage et de libération: en effet, les neurotransmetteurs «classiques» sont synthétisés dans le cytoplasme des neurones, puis stockés dans des vésicules synaptiques à partir desquelles ils sont libérés par exocytose dans la fente synaptique. Les endoCB sont synthétisés (dans les neurones et les astrocytes) «à la demande», après stimulation de différents récepteurs conduisant à l'hydrolyse de précurseurs lipidiques membranaires. De par leur nature lipidique, ils ne sont donc pas stockés dans des vésicules synaptiques, et diffusent librement après leur production [8].

Les récepteurs cannabinoïdes appartiennent à la famille des récepteurs à 7 domaines transmembranaires, groupe 1 de la famille $A$, et sont couplés à des protéines G. Le récepteur CB2 présente $44 \%$ d'homologie avec le CBl. Si le profil d'expression du CBl est extrêmement large (système nerveux central, tissus périphériques), celui du CB2 semble restreint aux cellules du système immunitaire. CBl est ainsi plutôt impliqué dans les effets psychotropes des $C B$, alors que CB2 l'est dans leurs effets immunomodulateurs. Le CB1 est l'un des récepteurs dont le taux d'expression est le
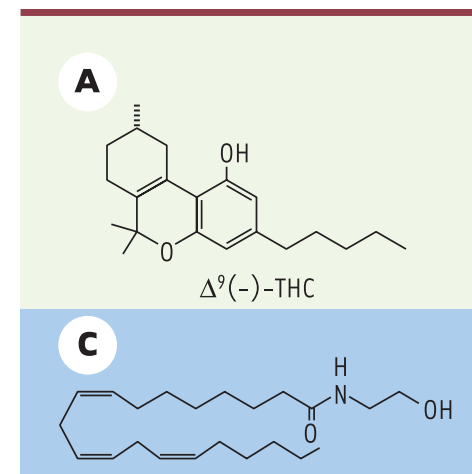

homo- $\gamma$-linolényl éthanol amide

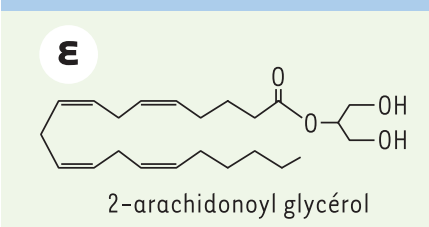

G

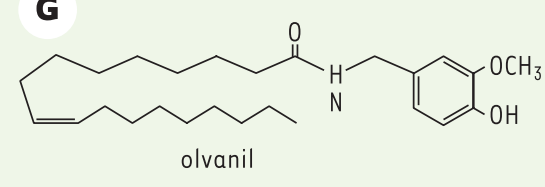

Figure 1. Structures chimiques $d u \Delta^{9}(-)-T H C(A)$, des endocannabinoïdes (endoCB): anandamide (AEA) (B), homo- $\gamma$-linolényl éthanol amide (C), docosatétraénoyl éthanol amide (D), 2-arachidonoyl glycérol (2AG) (E) et 2-arachidonoyl glycérol éther (F), ainsi que de l'olvanil (G), agoniste des récepteurs vanilloïdes VR1. Cinq endoCB ont été identifiés: les anandamides, trois éthanol amides dérivant d'acides gras différents, qui comprennent non seulement la «vraie» anandamide (AEA) (20:4, n-6, arachidonoyl éthanol amide) (B) [2], mais aussi le 22:4, n-6, docosatétraénoyl éthanol amide (D) et le 20:3, n-6, homo-linolényl éthanol amide (C) [5]. De ces trois composés, l'anandamide possède la meilleure efficacité pour les récepteurs CB. Le 2-AG est un ester de l'acide arachidonique ( $\varepsilon$ ) [6] et le 2-AG éther (noladin éther ou 2-AGE), un éther de l'acide arachidonique (F) [7]. Les concentrations d'AEA dans le cerveau sont comparables à celles de la dopamine ou de la sérotonine. Les principaux lieux de synthèse de l'AEA sont ceux dans lesquels a été montrée une forte expression du CBl. Contrairement à l'AEA, le 2-AG est un agoniste entier du récepteur $C B 2$. D'après des expériences de structure-activité, le $C B 2$ serait originellement le récepteur «2-AG». En fait, le 2-AG serait le «vrai» agoniste des récepteurs $C B 1$ et $C B 2$. L'AEA possède une affinité légèrement moindre pour le $C B 1$ et très faible pour le $C B 2$, ce qui expliquerait que l'AEA puisse activer d'autres récepteurs, tels que les récepteurs vanilloïdes, ce qui n'est pas le cas du 2-AG. Les concentrations de 2-AG dans le cerveau sont environ 170 fois supérieures à celles de l'AEA. 
plus élevé dans le SNC, mais quasiment absent du tronc cérébral, en accord avec l'absence de toxicité aiguë et de doses létales des dérivés du cannabis. Plusieurs études ont montré, pharmacologiquement [10] ou grâce à des lignées de souris $C B 1^{-/-}[11,12]$, qu'il devait exister au moins un autre récepteur $C B$.

Le métabolisme des endocannabinoïdes a surtout été étudié pour l'AEA et le 2-AG $[8,13]$. On peut noter que ce système endoCB semble être très ancien d'un point de vue phylogénétique, puisqu'il est présent des invertébrés jusqu'aux vertébrés, à l'exception notable des insectes.

\section{Endocannabinoïdes et voies de signalisation intracellulaire}

Les endoCB agissent principalement sur trois voies de signalisation intracellulaire: la modulation de l'adénylate cyclase, la modulation de la perméabilité de certains canaux ioniques et l'activation de la voie des protéine kinases activées par des agents mitogènes (MAP kinases) [14, 15] (Figure 2).

Les études ont montré que I'AEA active le récepteur VR1, alors que le 2-AG est incapable de s'y fixer [12]. Ce constat est capital pour l'orientation des recherches futures, puisque qu'il indique une quasi-dichotomie au sein des endoCB: le 2-AG serait le «véritable» endoCB, tandis que l'AEA serait un trans endoCB pouvant activer les $C B 1-2$, mais aussi d'autres récepteurs.

\section{Fonctions des endocannabinoïdes dans la neurotransmission: des messagers à contre-courant}

Les effets psychotropes des dérivés du cannabis sont dus à l'action de leurs principes actifs appelés phytoCB (dont le $\Delta^{9}-T H C$ ), qui prennent la place des endoCB sur les récepteurs CBI centraux [14]. Les zones de forte expression des récepteurs $C B 1$ sont les ganglions de la base, le cervelet (effets réversibles sur les performances psychomotrices et la coordination motrice), l'hippocampe (effets réversibles sur la mémoire à court terme et les fonctions cognitives) et le cortex, surtout au niveau des fibres et des terminaisons présynaptiques, plus faiblement dans les dendrites et les soma des neurones principaux. L'activation des CBI par les endoCB inhibe différents canaux calciques et module certains canaux potassiques présents à la fois sur les corps cellulaires et les prolongements axonaux [14]. Cela entraîne une réduction de la libération de neurotransmetteurs, de la durée du potentiel d'action et de la fréquence de décharge neuronale, à l'origine d'une mise sous silence transitoire (d'où l'absence d'effets neurotoxiques) des neurones exprimant les CB1.

Si l'activation des récepteurs CBl entraîne une inhibition de l'activité de certains neurones, cela ne signifie pas pour autant que les cannabinoïdes sont «inhibiteurs» des fonctions cérébrales. En raison d'effets de circuits, par exemple d'un effet de «désinhibition» par lequel les endoCB peuvent activer un circuit en inhibant des voies inhibitrices, les cannabinoïdes ont également la capacité, in fine, de provoquer l'excitation de populations neuronales ou de noyaux cérébraux [23].

Les endoCB sont capables, du fait de leur mode de production «à la demande» et de leurs propriétés chimiques particulières, d'intervenir «à contre-courant» de la transmission synaptique pour moduler de manière transitoire ou durable la libération de neurotransmetteurs [24, 25]. Dès 1991, l'équipe d'Alain Marty avait observé que la dépolarisation des cellules de Purkinje dans le cervelet entraînait une diminution, durant plusieurs dizaines de secondes, des événements postsynaptiques spontanés inhibiteurs GABAergiques (sIPSC) [26]. Ce nouveau phénomène, appelé DSI (depolarization induced suppression of inhibition), ne semblait pas d'origine postsynaptique (l'amplitude des SIPSC n'étant pas altérée lors du phénomène), mais présynaptique (la fréquence des sIPSC étant diminuée). Une DSI a également été rapportée au niveau des synapses GABAergiques de l'aire CAl de l'hippocampe [27]. Le messager rétrograde à l'origine de ce curieux mode de régulation de la présynapse par l'activité postsynaptique est resté inconnu jusqu'aux récents travaux de R.I. Wilson et R.A. Nicoll [24]: dans I'hippocampe, l'inhibition des récepteurs $C B I$ bloque la $D S I$, tandis que les $C B$ synthétiques entraînent l'occlusion de la DSI. Parallèlement, le rôle central des endoCB a également été révélé dans la DSE (depolarization induced suppression of excitation) du cervelet où la signalisation rétrograde endoCB module des synapses excitatrices glutamatergiques [25]. Ainsi, les endoCB sont des messagers rétrogrades qui, en réponse à une dépolarisation postsynaptique, réduisent pendant quelques dizaines de secondes la libération de neurotransmetteur, à contre-courant de la transmission synaptique inhibitrice ou excitatrice. L'étude pharmacologique des mécanismes de la DSI et de la DSE a révélé que, au niveau postsynaptique, la production d'endoCB implique de manière parallèle les récepteurs métabotropiques du glutamate (mGlu) et les canaux calciques [25] (Figure 3). L'efficacité des connexions synaptiques peut également, de manière durable (plusieurs heures in vitro, plusieurs jours in vivo), être modulée par leur propre activité. Ces formes élémentaires d'apprentissage sont regroupées sous le terme de plasticité synaptique à long terme. Fonctionnellement, la stimulation répétitive à haute fré- 
Figure 2. Principales voies de transduction du signal modifiées après action des endocannabinoïdes (endoCB) via les récepteurs CB1, VR1 ou par action directe. L'activation des récepteurs CB1 et CB2 par les endoCB (1) conduit à une inhibition de l'activité cyclasique (2). Cet effet est inhibé par un traitement par la toxine pertussique, révélant ainsi l'intervention d'une protéine $\mathrm{G}$ de type Gi/o dans le couplage récepteurenzyme [14, 15]. L'anandamide (AEA) et le 2-arachidonoyl glycérol (2-AG) sont des agonistes entiers de l'inhibition de l'adénylate cyclase via le CB1. En revanche, seul le 2-AG est agoniste entier au niveau du CB2. Dans certaines conditions, l'activation du récepteur CB1 (et pas le CB2) conduit à une production d'AMPc via une protéine de type Gs. Dans ce cas, l'AEA devient un agoniste partiel du CB1, contrairement au 2-AG qui reste agoniste entier. L'activation du récepteur CBl provoque également une inhibition indirecte, par le biais d'une protéine Gi/o mais indépendante de l'activité cyclasique, des canaux $\mathrm{Ca}^{2+}$ sensibles au potentiel de type N, L et $\mathrm{Q} / \mathrm{P}[14,15]$ (3), et une inhibition directe des canaux de type $T$ [16] (4). Les endoCB augmentent par ailleurs l'activité des canaux potassiques de la rectification entrante ( $\left.K_{I R}\right)(5)$, par le biais d'une protéine $\mathrm{G}$ de type Gi/o, mais indépendamment de l'inhibition de l'adénylate cyclase. L'activation des récepteurs CBI diminue la sensibilité au potentiel de membrane des canaux potassiques de type $A\left(K_{A}\right)(6)$, via une protéine Gi/o et de façon dépendante de l'inhibition de la voie adénylate cyclase/protéine kinase A (PKA) [14, 15]. De plus, les endoCB inhibent deux autres types de canaux potassiques: des canaux de fuite sensibles aux protons (TASK-1) et les canaux de type $M\left(K_{M}\right)[17,18](7)$. L'AEA a un double effet, inhibiteur et stimulateur, sur les récepteurs du glutamate de type NMDA (M-méthyl-D-aspartate) [14, 15]. L'inhibition est une conséquence indirecte de l'inhibition des conductances $\mathrm{Ca}^{2+}$ de type $P / Q$ après activation du $C B 1(8)$, tandis que l'activation résulte d'un effet direct des endoCB sur le récepteur NMDA (9), conduisant à une augmentation de l'influx calcique à travers le canal. L'AEA inhibe la perméabilité des jonctions communicantes entre les astrocytes, via une protéine $G$ de type $\mathrm{Gi} /$ o couplée à un récepteur de type $C B$ différent de $C B 1$; il a pour conséquence une inhibition du couplage électrique et de la propagation des vagues calciques intercellulaires [19]. Par ailleurs, les endoCB, après activation du récepteur $C B 1$ et activation subséquente de la phospholipase $C \beta(P L C \beta)$, stimulent la mobilisation du $\mathrm{Ca}^{2+}$ intracellulaire stocké dans le réticulum endoplasmique des neurones $[14,15]$ et des astrocytes (10) [20]. L'activation par les endoCB de la voie des MAP kinases (mitogen-activated protein kinases) $[14,15]$ (11) déclenche une cascade aboutissant in fine à l'activation de facteurs de transcription multiples, tels que krox 24, c-fos ou c-jun. Deux autres MAP kinases sont activées par les endoCB lors de stress cellulaires: $p 38-M A P K$ et $c$-jun- $N$-terminal kinase (JNK). L'activation de JNK conduit à une apoptose cellulaire, alors que celle de p38-MAPK a des effets neuroprotecteurs, ainsi qu'anti-profilérateurs au niveau de cellules tumorales [21, 22]. L'action des endoCB sur la voie des MAP kinases constitue un champ de recherche extrêmement prometteur au niveau thérapeutique (voir plus loin), car cette voie intervient dans le devenir de la cellule (processus de différenciation morphologique et de survie neuronale). Seule l'AEA, parmi les endoCB, active le récepteur vanilloïde de type 1 (VRl) (12), un canal cationique non sélectif de la famille des canaux TRP (transient receptor potential) impliqués dans les phénomènes de détection de stimulus nocicepteurs et dans la transduction de l'hyperalgésie inflammatoire et thermique. Un agoniste de VR1, l'olvanil (voir Figure 1), agit comme un agoniste partiel du $C B 1$, ce qui suggère l'existence d'un recouvrement partiel, dans la reconnaissance des ligands, entre les récepteurs VRI et CBI. Les affinités de couplage des récepteurs $C B 1$ et $C B 2$ aux protéines $G$ ne sont pas équivalentes: si les deux types de récepteurs possèdent une très forte affinité pour $\mathrm{Gi}$, celle du CBI pour Go est 10 fois supérieure à celle du CB2. Cela peut expliquer l'absence, ou la faiblesse, d'interaction du CB2 avec les canaux ioniques, contrairement au CBI qui est capable de moduler l'activité de nombreux canaux. Les endoCB inhibent, directement ou indirectement (via le $C B 1$ ), la perméabilité de canaux calciques préférentiellement présynaptiques (comme les récepteurs CBI) et impliqués dans le contrôle de la libération des neurotransmetteurs.

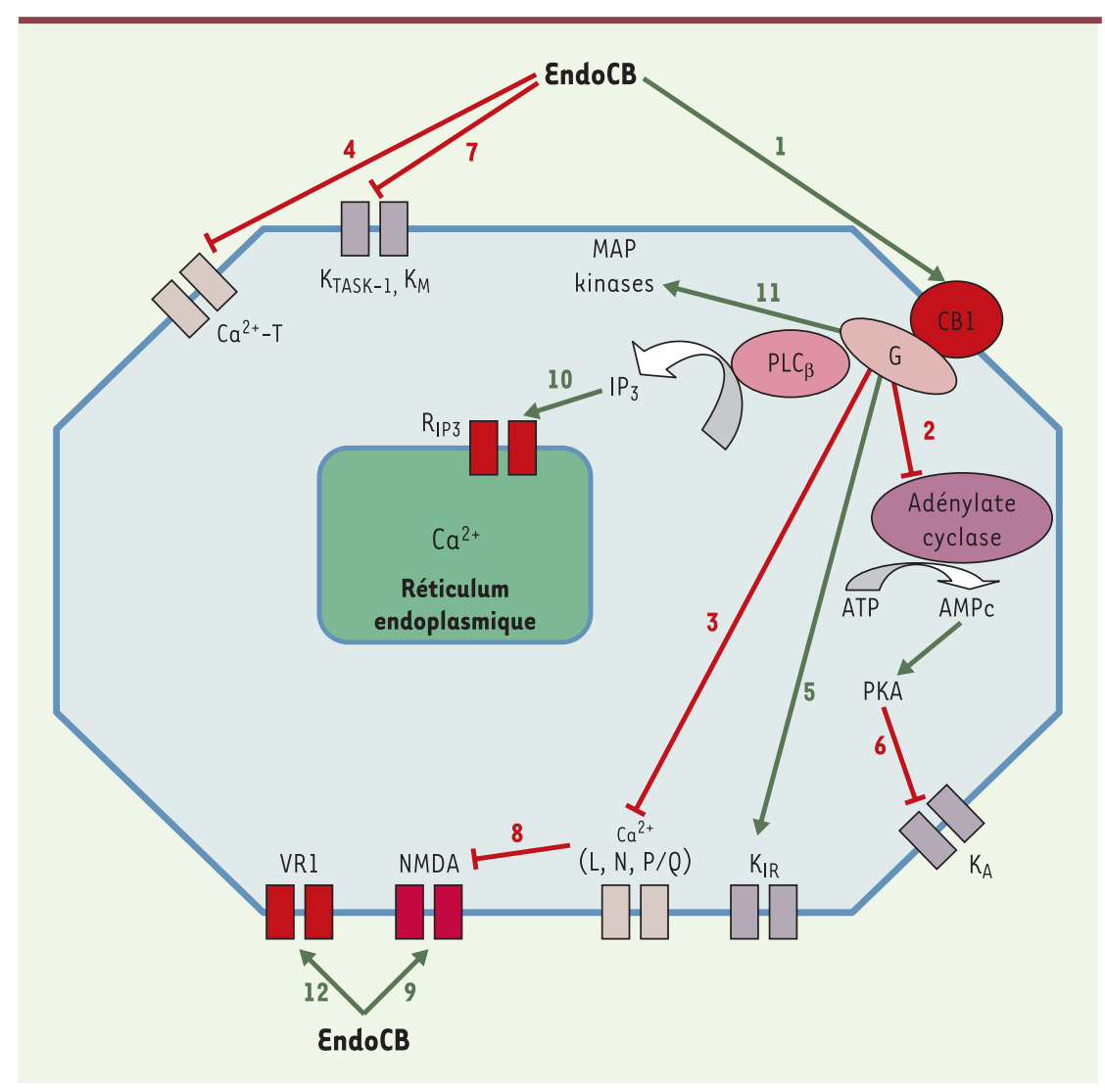


quence (autour de $100 \mathrm{~Hz}$ ) induit une potentialisation durable de la transmission synaptique (à intensité de stimulation présynaptique égale, la réponse postsynaptique est plus grande), nommée potentialisation à long terme. D'autres modes de stimulation (à basse fréquence) induisent au contraire une dépression à long terme (DLT) pouvant durer plusieurs heures in vitro. Ces deux formes archétypiques ont été observées au niveau de très nombreuses synapses excitatrices du SNC. Or, les endoCB pourraient moduler la plasticité synaptique à long terme, comme cela a été montré au niveau du noyau accumbens, structure centrale de la voie corticomésolimbique. Le noyau accumbens reçoit, entre autres, des afférences glutamatergiques du cortex préfrontal. La stimulation de ces fibres excitatrices, à une fréquence correspondant aux rythmes de décharge, mesurés in vivo, des neurones du cortex prélimbique ( $13 \mathrm{~Hz}, 10$ minutes), entraîne une DLT importante de l'efficacité de la transmission excitatrice corticale afférente au noyau accumbens. Les récepteurs $\mathrm{CBl}$ étant présents sur les terminaisons en provenance du cortex prélimbique [28], et compte tenu du rôle de la voie de signalisation rétrograde endoCB dans la DSI et la DSE, il est possible que les endoCB contrôlent la plasticité synaptique à long terme. Corroborant cette hypothèse, cette DLT est absente chez des souris $C B 1^{-/-}$, et bloquée par un antagoniste $\mathrm{CBl}$ chez des souris sauvages $[29,30]$. Des résultats similaires ont été obtenus au niveau des synapses corticostriatales [31].

Les endoCB sont des messagers rétrogrades qui diffusent au-delà des synapses glutamatergiques à l'origine de leur production pour agir sur les synapses environnantes. Ainsi, la stimulation à haute fréquence de l'amygdale basolatérale entraîne une DLT des synapses GABAergiques voisines [32], qui pourrait être associée à l'extinction des mémoires d'aversion. Les endoCB interviendraient donc dans la plasticité synaptique «en volume», c'est-à-dire en réglant l'efficacité synaptique de terminaisons neuronales voisines des synapses, lieux de leur production. Les endoCB représentent donc une nouvelle classe de messagers diffusibles impliqués dans les régulations à court et à long terme de la transmission synaptique $[24,25,30]$.

\section{Effets comportementaux des endocannabinoïdes}

Les endoCB produisent des effets somatiques similaires à ceux induits par les phytoCB [33]. Ces effets incluent chez le rongeur des réponses bien définies: antinociception, hypothermie, hypolocomotion et catalepsie. Les doses faibles produisent chez l'animal des réponses comportementales qui sont un mélange des effets stimulateurs et dépresseurs des CB sur le SNC. Cependant, à doses élevées, les effets des CB sont toujours de type dépresseur [34].

\section{Effets moteurs}

Des doses élevées de CB induisent chez la souris un comportement pop corn: les souris restent dans un état de sédation apparente, mais bondissent (hyperréflexie) lors de stimulus tactiles ou auditifs. Chez le rat, des doses élevées de $C B$ induisent un comportement de rotation autour du corps [33-35]. Dans le striatum, les endoCB jouent un rôle inhibiteur sur le contrôle de la motricité qui s'opposerait aux effets facilitateurs de la dopamine. Par ailleurs, le cervelet participe lui aussi à certaines actions motrices des CB, comme l'ataxie et la perte de coordination.

\section{Effets antinociceptifs}

Les CB ont des effets antinociceptifs dans différents modèles animaux, et l'antagoniste CBI SR141716A induit des effets hyperalgiques qui suggèrent l'existence d'un tonus endoCB analgésique [36]. Les endoCB modulent la nociception par des mécanismes supraspinaux, spinaux et périphériques. La micro-injection centrale de CB a permis d'identifier différentes régions responsables de ces réponses, telles que la substance grise périaqueducale ou la partie rostroventromédiane du bulbe rachidien. Une partie des effets antinociceptifs centraux semble due à la modulation de l'activité du système inhibiteur descendant. Au niveau spinal, les CB sont efficaces pour inhiber la transmission des fibres nociceptives de petit diamètre, et ils diminueraient la libération de neurotransmetteurs tels que la substance $P$ ou le calcitonin gene-related peptide, responsables de la transmission de la douleur [36]. De plus, les récepteurs vanilloïdes participeraient à ces réponses antinociceptives [37]. Enfin, au niveau périphérique, les récepteurs $C B 1$ et $C B 2$ jouent un rôle synergique d'inhibition des stimulus nociceptifs [38]: une libération d'AEA et de palmityl éthanolamine a ainsi été démontrée dans des modèles de douleurs d'origine inflammatoire.

\section{Effets sur la mémoire}

Les $C B$, via les $C B 1$, diminuent l'acquisition des apprentissages et la mémoire de travail, mais n'ont pas d'effet sur la mémoire de référence. L'hippocampe serait la principale structure responsable des effets des CB sur la mémoire: ainsi, les CB diminuent la potentialisation et la dépression à long terme observées dans les neurones de I'hippocampe [39]. Les endoCB sont libérés par la stimulation des neurones hippocampiques, suggérant un rôle tonique important dans leur contrôle physiolo- 
gique de la mémoire [40]. En accord avec ces résultats, les souris $\mathrm{CBI}^{-/-}$semblent se comporter mieux que leurs congénères sauvages dans un test de mémorisation hippocampique [4l].

\section{Autres réponses comportementales}

Les CB augmentent le sommeil par l'intermédiaire d'un lipide, l'oléamide (augmentation des phases lente et paradoxale REM, rapid eye movements), avec pour corollaire une diminution du temps d'éveil [42]. De plus, l'antagoniste CBI SR141716A augmente l'état d'éveil, suggérant un rôle physiologique du système endoCB dans le contrôle des états de sommeil et de vigilance.

Les effets des CB sur l'anxiété sont biphasiques. Des effets anxiogènes ont été observés après l'administration de doses élevées de différents agonistes CB [43], tandis que des doses faibles induisent des effets anxiolytiques [44]. Par ailleurs, l'antagoniste CBI SR141716A induit des effets anxiogènes, ce qui suggère que le blocage du tonus endoCB augmente l'anxiété. Des effets opposés des CB ont également été décrits concernant l'agressivité : l'administration chronique de doses élevées du principe actif des cannabinoïdes, le THC, induit une augmentation de l'agressivité chez le rat; en revanche, des doses faibles de THC, plus proches des doses éventuellement consommées par l'homme via le cannabis, induisent une diminution de l'agressivité [45].

Enfin, un lipide de la même famille que les endoCB, l'oléyléthanolamide, est impliqué dans le contrôle physiologique de la prise alimentaire [46].

\section{Potentiel thérapeutique du cannabis ou de ses dérivés: un remède vieux comme le monde}

Les premières traces d'un usage médicinal des dérivés du cannabis sont retrouvées dans des textes chinois et égyptiens datés de plusieurs centaines d'années avant J.-C. Les phytoCB sont utilisés depuis des millénaires pour traiter la douleur, les spasmes, les nausées, l'insomnie ou le manque d'appétit. après induction de la DLT.

\section{Traitement de la douleur}

Les phyto $C B$ et les endoCB se sont révélés très efficaces (parfois plus que les opiacés) dans des modèles animaux de douleurs aiguës, inflammatoires, neuropathiques et d'hyperalgie $[36,47]$, et le pouvoir antinociceptif des agonistes CBI a également été observé chez l'homme [48]. Le fait que les endoCB agissent, dans certains cas, de manière indépendante des récepteurs classiques des CB au niveau central permet d'envisager le développement de molécules thérapeutiques

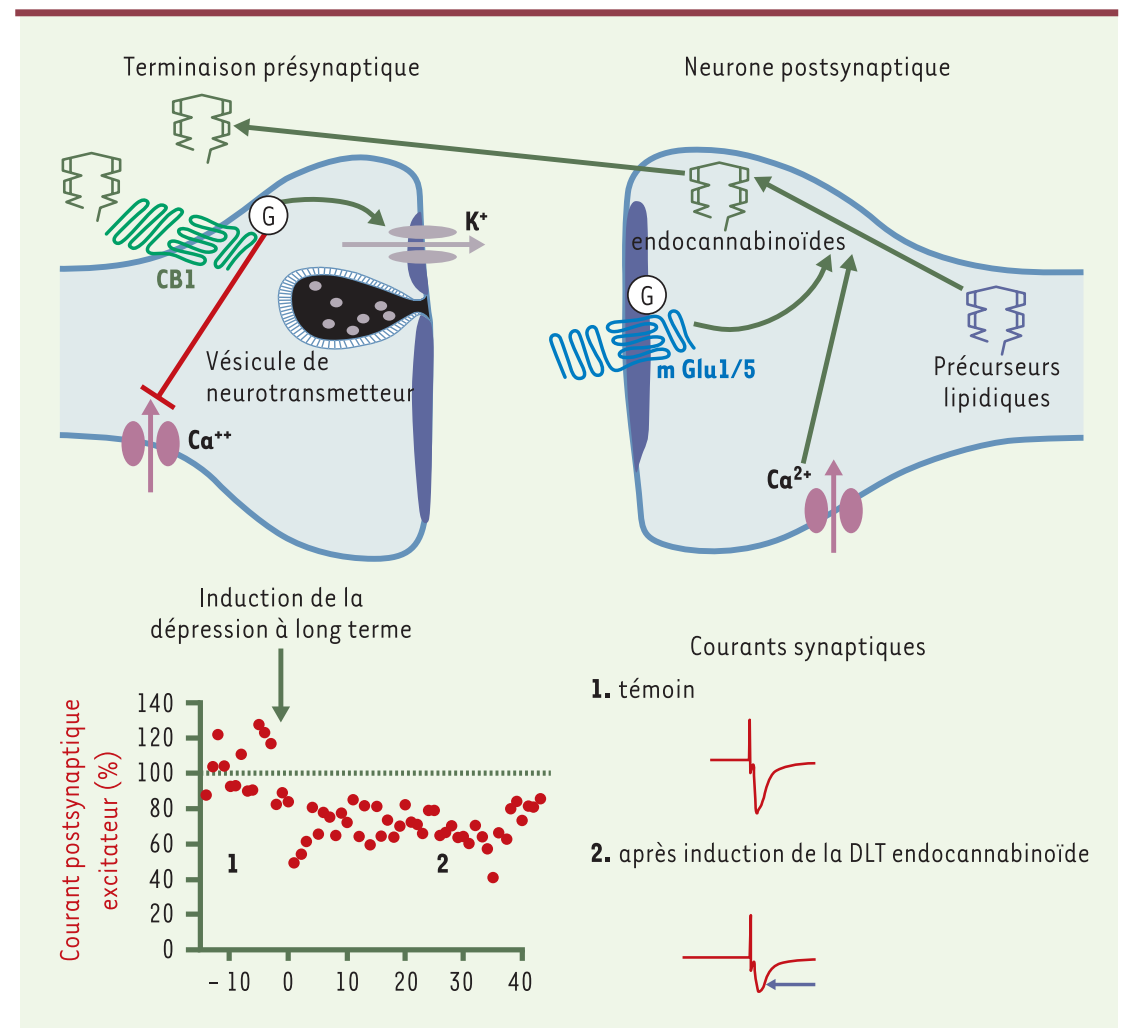

Figure 3. Les endocannabinoïdes (endoCB) agissent sur la plasticité synaptique à long terme, grâce à leur action rétrograde sur l'élément présynaptique. En haut: représentation schématique de la voie rétrograde de signalisation endoCB. L'activation postsynaptique des récepteurs $\mathrm{mGlul} / 5$ et/ou l'élévation du $\mathrm{Ca}^{2+}$ intracellulaire postsynaptique entraîne la production d'endoCB et l'activation de récepteurs $C B 1$ présynaptiques. Ce mécanisme général est valable pour les effets à court terme (DSI, depolarization induced suppression of inhibition; DSE, depolarization induced suppression of excitation) comme à long terme (DLT, dépression à long terme) des endoCB. Le glutamate libéré lors de la stimulation soutenue des fibres prélimbiques active au niveau postsynaptique des récepteurs du glutamate de type mGlu5, avec pour effet une vidange des compartiments calciques intracellulaires, ainsi que la production et la libération d'endoCB par le neurone postsynaptique. Ces endoCB agissent in fine sur les récepteurs CB1 présents sur les terminaisons présynaptiques pour diminuer de manière durable la libération de glutamate [29]. Ce phénomène permet donc de traduire (via les récepteurs mGlu5) le signal glutamatergique antérograde «point à point» rapide en signal endoCB rétrograde agissant à long terme [30]. En bas: enregistrements de la transmission synaptique glutamatergique dans le noyau accumbens de souris. Les courants postsynaptiques excitateurs sont fortement réduits 
sans effets psychotropes. Par ailleurs, puisque les récepteurs $C B 1$ et $C B 2$ semblent tous deux impliqués dans l'effet antinociceptif au niveau périphérique, il existe une possibilité de développer des agents thérapeutiques spécifiques des CB2, efficaces sur la douleur mais dépourvus d'effets psychotropes.

\section{Traitement de glaucomes}

Les dérivés du cannabis sont depuis longtemps utilisés pour traiter les glaucomes, l'activation des récepteurs $\mathrm{CBl}$ causant une vasodilatation et une réduction de la pression intra-oculaire [49]. II est probable que la découverte récente de récepteurs $C B 1$ et du système endoCB dans l'œil permette le développement de préparations à application locale, permettant ainsi d'éviter les effets psychoactifs des CBI [49].

\section{Traitement de désordres}

\section{du comportement alimentaire}

Les phytoCB et les endoCB stimulent l'appétit, tandis que les antagonistes $C B I$ sont anorexigènes et entrấnent une perte de poids chez les souris obèses [50]. De plus, le 2-AG présent dans le lait maternel stimule la tétée, le blocage des récepteurs $\mathrm{CBl}$ chez les souriceaux nouveau-nés entraînant un arrêt de la prise de lait et la mort des animaux [4]. Ces propriétés laissent présager du potentiel thérapeutique des agents pharmacologiques $C B$ et endoCB dans le traitement de désordres aussi importants que l'obésité ou l'anorexie.

Par ailleurs, les caractéristiques anti-émétiques bien connues des CB s'ajoutent à leurs effets orexigènes et expliquent les résultats encourageants obtenus avec des agonistes CBl utilisés dans le traitement des effets secondaires particulièrement dévastateurs des chimiothérapies anticancéreuses et des pertes de poids accompagnant l'infection par le VIH [4, 50].

\section{Traitement de désordres moteurs}

Au niveau des ganglions de la base, la stimulation des récepteurs $C B 1$ diminuerait les symptômes d'hyperactivité dopaminergique liés à de nombreuses maladies neuropsychiatriques. Ainsi, les CB présenteraient un intérêt thérapeutique dans le traitement des tics accompagnant le syndrome de Gilles de la Tourette, dans la réduction des dyskinésies induites par le traitement lévodopa des parkinsoniens et dans certaines formes de tremblements et de dystonies [51, 52]. Notons enfin les résultats significatifs obtenus dans le traitement des tremblements et des spasmes chez les malades atteints de sclérose en plaques, ainsi que dans un modèle animal auto-immun de sclérose en plaques [53].

\section{Traitement d'ischémies cérébrales}

II n'existe pas de traitement approprié aux accidents cérébraux d'origine traumatique ou ischémique. Une stratégie proposée consisterait à contrer le principal mécanisme secondaire, l'excitotoxicité glutamatergique, en agissant sur les voies neuroprotectrices et réparatrices endogènes. La capacité des endoCB à réduire la libération de glutamate jouerait un rôle important dans le fort pouvoir neuroprotecteur des phytoCB et des endoCB observé dans des modèles ani$\operatorname{maux}[51,54]$.

\section{Traitement de gliomes}

C'est là une des potentialités thérapeutique les plus prometteuses des CB, puisque les gliomes sont jusqu'à présent des maladies au pronostic très réservé. Les $C B$ participent au contrôle du devenir cellulaire, survie ou mort, via l'induction de la synthèse de céramide. On sait que des concentrations élevées et soutenues de céramide induisent une apoptose cellulaire, alors que des concentrations élevées mais brèves favorisent au contraire la régulation de fonctions métaboliques. Or, dans plusieurs types de gliomes, l'activation des récepteurs $C B$ conduit à des concentrations chroniquement élevées de céramide et donc à l'apoptose de ces cellules tumorales. Cet effet est propre aux gliomes, l'activation même soutenue des récepteurs CB astrocytaires ou neuronaux entraînant des élévations aiguës (et non chroniques) de céramide, n'ayant ainsi aucun effet apoptotique sur ces cellules $[21,22]$.

\section{Conclusions}

L'étude du système endocannabinoïde est fascinante par son intérêt propre, qui dépasse très largement les effets des cannabinoïdes exogènes, mais aussi par I'histoire de son étude. Celle-ci a en effet été jalonnée de rebondissements, qui ont souvent pris en défaut la logique scientifique, mais qui ont abouti à la mise en évidence d'un système endocannabinoïde extrêmement complexe. Les progrès récents réalisés dans la compréhension des effets cellulaires et moléculaires des endocannabinoïdes, ainsi que dans le développement d'agents pharmacologiques sélectifs de leurs différentes voies de synthèse et de dégradation, suggèrent que ces molécules agissent de manière extrêmement fine sur de multiples voies de transduction du signal et de l'information. Le système endocannabinoïde apparaît dès lors comme un formidable terrain de recherche pour le développement de nouvelles thérapeutiques. $\diamond$ 


\section{SUMMARY}

Endocannabinoids in the central nervous system

The major psychoactive component of cannabis derivatives, $\Delta^{9}-T H C$, activates two G-protein coupled receptors: $C B 1$ and $C B 2$. Soon after the discovery of these receptors, their endogenous ligands were identified: lipid metabolites of arachidonic acid, named endocannabinoids. The two major main and most studied endocannabinoids are anandamide and 2-arachidonyl-glycerol. The CBI receptor is massively expressed throughout the central nervous system whereas CB2 expression seems restricted to immune cells. Following endocannabinoid binding, $\mathrm{CBl}$ receptors modulate second messenger cascades (inhibition of adenylate cyclase, activation of mitogen-activated protein kinases and of focaladhesion kinases) as well as ionic conductances (inhibition of voltage-dependent calcium channels, activation of several potassium channels). Endocannabinoids transiently silence synapses by decreasing neurotransmitter release, play major parts in various forms of synaptic plasticity because of their ability to behave as retrograde messengers and activate non-cannabinoid receptors (such as vanilloid receptor type-1), illustrating the complexity of the endocannabinoid system. The diverse cellular targets of endocannabinoids are at the origin of the promising therapeutic potentials of the endocannabinoid system. $\diamond$

\section{RÉFÉRENCES}

1. Matsuda LA, Lolait SJ, Brownstein MJ, Young AC, Bonner T. Structure of a cannabinoid receptor and functional expression of the cloned cDNA. Nature 1990; 346: 561-4.

2. Devane WA, Hanus L, Breuer $A$, et al. Isolation and structure of a brain constituent that binds to the cannabinoid receptor. Science 1992; 258: 1946-9.

3. Munro S, Thomas KL, AbuShaar M. Molecular characterization of a peripheral receptor for cannabinoids. Nature 1993; 365: 61-5.

4. Fride $\varepsilon$. Endocannabinoids in the central nervous system-an overview. Prostaglandins, Leukotrienes and Essential Fatty Acids 2002; $66: 221-33$.
8. Giuffrida R, Beltramo M, Piomelli D. Mechanism of endocannabinoid inactivation: biochemistry and pharmacology. J Pharm Exp Ther 2001; 298: 7-14.

9. Pertwee RG, Ross RA. Cannabinoid receptors and their ligands.

Prostaglandins, Leukotrienes and Essential Fatty Acids 2002; 66: 101-21.

10. Sagan S, Venance L, Torrens y, Cordier J, Glowinski J, Giaume C. In cultured mouse astrocytes, but not in neurons, anandamide has pharmacological effects distincts from synthetic cannabinoids. Eur J Neurosci 1999; 11: 691-9.

11. Haller J, Bakos N, Szirmay M, Ledent C, Freund TF. The effects of genetic and pharmacological blockade of the CBI cannabinoid receptors on anxiety. Eur J Neurosci 2002; 16: 395-8.

12. Di Marzo V, De Petrocellis $L$, Fezza F, Ligresti A, Bisogno T. Anandamide receptors. Prostaglandins, Leukotrienes and Essential Fatty Acids 2002; 66: 377-91.

13. Suguira T, Koyabashi Y, Oka $S$, Waku K. Biosynthesis and degradation of anandamide and 2-arachidonoylglycerol and their possible physiological significance. Prostaglandins, Leukotrienes and Essential Fatty Acids 2002; 66: 173-92.

14. Ameri A. The effects of cannabinoids on the brain. Prog Neurobiol 1999; 58: 315-48.

15. McAllister SD, Glass M. CBI and $C B 2$ receptor-mediated signalling: a focus on endocannabinoids. Prostaglandins, Leukotrienes and Essential Fatty Acids 2002; 66: 161-71.
16. Chemin J, Monteil A, PerezReyes $\varepsilon$, Nargeot J, Lory P. Direct inhibition of T-type calcium channels by the endogenous cannabinoid anandamide. EMBO J 2001; 20: $7033-40$.

17. Maingret $F$, Patel AJ, Lazdunski M, Honore $\varepsilon$. The endocannabinoid anandamide is a direct and selective blocker of the background $\mathrm{K}^{+}$channel TASK-1. EMBO J 2001; 20: 47-54.

18. Schweitzer P. Cannabinoids decrease the $K^{+} M$-current in hippocampal CAl neurons. J Neurosci 2000; 20: 51-8.

19. Venance L, Piomelli D, Glowinski J, Giaume C. Inhibition by anadamide of gap junctions and intercellular calcium signalling in striatal astrocytes. Nature 1995; 376: 590-4.

20. Venance L, Sagan S, Giaume C. R-methanandamide inhibits receptor-induced calcium responses by depleting internal calcium stores in cultured astrocytes. Eur J Physiol 1997; 434: 147-9.

21. Galve-Roperh I, Sanchez C, Cortes ML, Gomez del Pulgar T, Izquierdo M, Guzman M. Anti-tumoral action of cannabinoids: involvement of sustained ceramide accumulation and extracellular signalregulated kinase regulation. Nat Med 2000 ; 6 : 313-9.

22. Guzman M, Sanchez C, Galve-Roperh I. Cannabinoids and cell fate. Pharmacol Ther 2002; 95: 175-84.

23. Carlson $G$, Wang $Y$, Alger BE. Endocannabinoids facilitate the induction of LTP in the hippocampus. Nat Neurosci 2002; 5 : 723-4.

24. Wilson RI, Nicoll RA. Endocannabinoid signaling in the brain. Science 2002; 296: 678-82. 
25. Kreitzer AC, Regehr WG. Retrograde signaling by endocannabinoids. Curr Opin Neurobiol 2002; 12: 324-30.

26. Llano I, Leresche N, Marty A. Calcium entry increases the sensitivity of cerebellar Purkinje cells to applied GABA and decreases inhibitory synaptic currents. Neuron 1991; 6: 565-74.

27. Pitler TA, Alger BE. Postsynaptic spike firing reduces synaptic GABAA responses in hippocampal pyramidal cells. J Neurosci 1992; 12: 4122-32.

28. Robbe D, Alonso G, Duchamp F, Bockaert J, Manzoni 0J. Localization and mechanisms of action of cannabinoid receptors at the glutamatergic synapses of the mouse nucleus accumbens. J Neurosci 2001; 21: 109-16.

29. Robbe D, Kopf M, Remaury A, Bockaert J, Manzoni 0J. Endogenous cannabinoids mediate long-term synaptic depression in the nucleus accumbens. Proc Natl Acad Sci USA 2002; 99 : 8384-8.

30. Doherty J, Dingledine R. Functional interactions between cannabinoid and metabotropic glutamate receptors in the central nervous system. Curr Opin Pharmacol 2003; 3: 46-53.

31. Gerdeman GL, Ronesi J, Lovinger DM. Postsynaptic endocannabinoid release is critical to long-term depression in the striatum. Nat Neurosci 2002; 5: 446-51.

32. Marsicano G, Wotjak CT, Azad SC, et al. The endogenous cannabinoid system controls extinction of aversive memories. Nature 2002; 418: 530-4.
33. Costa B, Vailati S, Colleoni M. SR 141716A, a cannabinoid receptor antagonist, reverses the behavioural effects of anandamide-treated rats. Behav Pharmacol 1999; 10: 327-31.

34. Dewey WL. Cannabinoid pharmacology. Pharmacol Rev 1986; 38: 151-78.

35. Ledent C, Valverde 0, Cossu $\mathrm{G}$, et al. Unresponsivenes to cannabinoids and feduced addictive effects of opiates in $\mathrm{CB} 1$ receptor knockout mice. Science 1999; 283: 15.

36. Pertwee RG. Cannabinoid receptors and pain. Prog Neurobiol 2001; 63: 569-611.

37. Welch SP, Huffman JW, Lowe J. Differential blockade of the antinociceptive effects of centrally administered cannabinoids by SR141716A. J Pharmacol Exp Ther 1998; 286: 1301-8.

38. Calignano A, La Rana G, Giuffrida A, Piomelli D. Control of pain initiation by endogenous cannabinoids. Nature 1998; 394: 277-281.

39. Misner DL, Sullivan JM. Mechanism of cannabinoid effects on long-term potentiation and depression in hippocampal CAl neurons. J Neurosci 1999; 19: 6795-805.

40. Stella N, Schweitzer P, Piomelli D. A second endogenous cannabinoid that modulates long-term potentiation. Nature 1997; 388: 773-8.
41. Reibaud M, Obinu MC, Ledent C, Parmentier M, Bohme GA, Imperato A. Enhancement of memory in cannabinoid $\mathrm{CB} l$ receptor knock-out mice. EurJ Pharmacol 1999; 379: R1-R2

42. Murillo-Rodriguez $\varepsilon$, Sanchez-Alavez M, Navarro L, Martinez-Gonzalez D, Drucker-Colin R, ProsperoGarcia 0. Anandamide modulates sleep and memory in rats. Brain Res 1998; 812: 270-4.

43. Chakrabarti A, Ekuta JE, Onaivi ES. Neurobehavioral effects of anandamide and cannabinoid receptor gene expression in mice. Brain Res Bull 1998; 45: 67-74

44. Berrendero F, Maldonado R. Involvement of the opioid system in the anxiolytic-like effects induced by $\Delta^{9}$ tetrahydrocannabinol. Psychopharmacology 2002; 163: 111-7.

45. Miczek KA. Delta9tetrahydrocannabinol: Antiagressive effects in mice, rats, and squirrel monkeys. Science 1978; 199: 1459-61.

46. Rodriguez de Fonseca $F$, Navarro M, Gómez R, et al. An anorexic lipid mediator regulated by feeding. Nature 2001; 414: 209-12.

47. Walker JM, Huang SM. Cannabinoid analgesia. Pharmacol Ther 2002; 95: 127-35.
48. Hamann W, di Vadi PP. Analgesic effect of the cannabinoid analogue nabilone is not mediated by opioid receptors. Lancet 1999; 353: 560.

49. Jarvinen T, Pate DW, Laine K. Cannabinoids in the treatment of glaucoma. Pharmacol Ther 2002; 95: 203-20.

50. Berry EM, Mechoulam R. Tetrahydrocannabinol and endocannabinoids in feeding and appetite. Pharmacol Ther 2002; 95: 185-90.

51. Piomelli D, Giuffrida A, Calignano A, Rodriguez de Fonseca F. The endocannabinoid system as a target for therapeutic drugs. Trends Pharmacol Sci 2000; $21: 218-24$.

52. Romero J, Lastres-Becker I, de Miguel R, et al. The endogenous cannabinoid system and the basal ganglia. Biochemical, pharmacological, and therapeutic aspects. Pharmacol Ther 2002; 95: 137-52.

53. Pertwee RG. Cannabinoids and multiple sclerosis. Pharmacol Ther 2002; 95: 165-74.

54. Van der Stelt M, Veldhuis WB, Maccarrone M, et al. Acute neuronal injury, excitotoxicity, and the endocannabinoid system. Mol Neurobiol 2002; 26: 317-46.
TIRÉS À PART

L. Venance 